including the TUG test. TUG performance was considered low if it took, 15 seconds or longer. A vertebral fracture assessment (VFA) image was obtained which was subsequently scored for the presence of significant vertebral deformities. VF grade 1 were excluded.

Results: Eighty four patients were included. The mean age of our patients was $62.29 \pm 8.27$ years with an average body mass index (BMI) of $31.45 \pm 6.21 \mathrm{~kg} / \mathrm{m}^{2}$ [18.18-44.38]. According to the WHO classification 42 patients $(50 \%)$ had osteoporosis. Among 84 women, 47 had a low TUG performance. Subjects with low TUG performance had an older age (57.94 vs 65.71 years) $(p<0.001)$, and had a significantly lower BMD at femoral neck $\left(0.825 \mathrm{~g} / \mathrm{cm}^{3} v s \quad 0.752 \mathrm{~g} / \mathrm{cm}^{3}\right) \quad(p<0.001)$. There was no significant difference between the two groups regarding duration of menopause, history of previous fragility fracture, body mass index and BMD at total hip and at vertebral site. Osteoporosis was found in 30 patients who had low TUG performance and in 12 patients who had normal TUG performance without statistically significant difference $(p=0.08)$.VF were assessed in 22 subjects. VF were found in 20 patients with low TUG performance $(42.55 \%)$. Compared with the group with normal TUG performance, subjects with low TUG performance had significantly higher number of fracture $(p<0.001)$.A significant correlation was found between the number of VF and the score of get up and go test (Khi-2 test, $\mathrm{p}<0.001)$.

Conclusion: Our study found a strong relationship between TUG performance and prevalent vertebral fracture. Therefore evaluation of additional risk factors may improve the prediction of vertebral fracture in post-menopausal women.

Disclosure of Interests: None declared

DOI: 10.1136/annrheumdis-2019-eular.6377

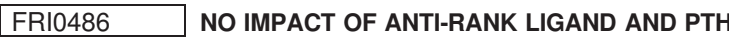 ANALOGUES ON CARDIOVASCULAR RISK IN IDIOPATHIC AND POSTMENOPAUSAL OSTEOPOROSIS: A SYSTEMATIC LITERATURE REVIEW AND META- ANALYSIS}

Laurence Ferrieres, Yannick Degboe, Michel Laroche, Arnaud Constantin, Adeline Ruyssen-Witrand. Hospital Center University Toulouse - Casselardit Ancely, Toulouse, France

Background: Emerging evidence suggests a possible association between osteoporosis and cardiovascular disease ${ }^{1,2}$. The mutual effects of drugs used in these two diseases are now a point of interest. Two meta-analyses have been conducted to explore the cardiovascular effects of bisphosphonates $^{3,4}$. There is no review for other osteoporosis treatments, such as anti-rank ligand antibody and parathyroid hormone analogues.

Objectives: A literature review and meta-analysis of randomized controlled trials was conducted to address the impact of PTH analogues and antiRank Ligand on cardiovascular events and overall mortality in individuals with idiopathic osteoporosis

Methods: A systematic literature review was conducted in December 2017 in the PubMed, Embase, Cochrane databases, and updated on PubMed in December 2018, by selecting trials including a treatment (antiRank Ligand or PTH analogue) and a control group. We also conducted a search for abstracts of the French Rheumatology Society, American College of Rheumatology and European League Against Rheumatisms annual meetings over the past 3 years. Relative risks and 95\% confidence intervals for each event were estimated using the Inverse Variance fixed effects method. The main endpoints were the occurrence of cardiovascular events and all-cause of mortality.

Results: Of the 2782 reports initially found (503 articles via PubMed, 1309 via Embase, 474 via the Cochrane Library and 15 abstracts from annual meetings, 16 articles were used for the meta-analysis. All studies were randomized; no exploitable prospective cohort study was found. The number of adverse events of interest was available for 6 studies for the anti-Rank Ligand corresponding to 26864 patient-years. After meta-analysis, there was no significant difference between the placebo group and the anti-Rank Ligand group for overall mortality (RR $0.79,95 \% \mathrm{Cl}(0.60$ 1.04)), the combined cardiovascular endpoint (overall mortality, coronary artery disease and stroke: $\mathrm{RR} 0.95,95 \% \mathrm{Cl}(0.78-1.16))$, and the individual risk of coronary artery disease (RR 1.14, 95\% Cl (0.76-1.73)), arrhythmia (RR 1.03, 95\% Cl $(0.63-1.68)$ ) and ischemic or hemorrhagic stroke (RR $1.03,95 \% \mathrm{Cl}(0.72-1.47)$ ). The number of adverse events of interest was available for 10 studies for the PTH analogue group, corresponding to 6220 patient-years. After meta-analysis, there was no significant difference between the placebo group and the PTH analogue group for overall mortality ( $\mathrm{RR} 0.89,95 \% \mathrm{Cl}(0.35-2.29)$ ), the combined endpoint (overall mortality, coronary artery disease and stroke: RR 0.71 , 95\% $\mathrm{Cl}(0.37-1.36)$ ), and the individual risk of coronary artery disease (RR
$0.65,95 \% \mathrm{Cl}(0.19-2.27)$ ), arrhythmia disorders (RR 1.43, 95\% Cl (0.73$2.80)$ ) and ischemic or hemorrhagic stroke (RR $0.60,95 \% \mathrm{Cl}(0.19-1.96)$ ). Conclusion: The anti-Rank Ligand and the PTH analogues have no short-term impact on the cardiovascular risk and the overall mortality in postmenopausal osteoporosis. To better answer the question whether these treatments are able to reduce the long-term cardiovascular risk, further comparative studies with longer duration are required.

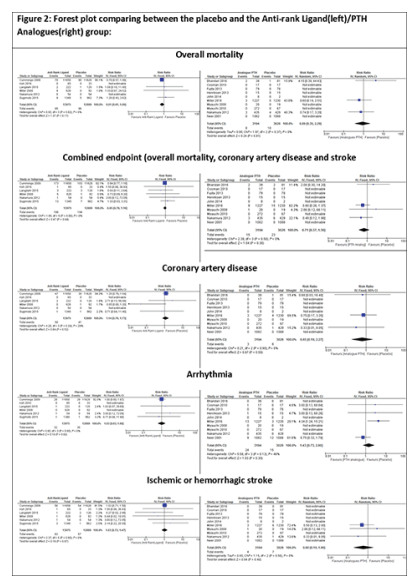

REFERENCES:

[1] von der Recke P, et al. The association between low bone mass at the menopause and cardiovascular mortality. Am J Med. 1999;106(3):273-278.

[2] Veronese $\mathrm{N}$, et al. Relationship Between Low Bone Mineral Density and Fractures With Incident Cardiovascular Disease: A Systematic Review and Meta-Analysis. J Bone Miner Res Off J Am Soc Bone Miner Res. 2017;32 (5):1126-1135

[3] Kim DH, et al. Bisphosphonates and Risk of Cardiovascular Events: A Meta-Analysis. Pizzi C, ed. PLOS ONE. 2015;10(4):e0122646.

[4] Kranenburg $G$, et al. Bisphosphonates for cardiovascular risk reduction: A systematic review and meta-analysis. Atherosclerosis. 2016;252:106-115.

Disclosure of Interests: None declared

DOI: 10.1136/annrheumdis-2019-eular.2032

\section{FRI0487 UTILITY OF TRABECULAR BONE SCORE(TBS) FOR FRACTURE RISK ASSESSMENT IN GLUCOCORTICOID- INDUCED OSTEOPOROSIS}

Helena Florez ${ }^{1}$, José Hernández-Rodríguez ${ }^{2}$, Africa Muxi ${ }^{3}$, Josep Lluís Carrasco ${ }^{4}$, Sergio Prieto-González ${ }^{2}$, Silvia Ruiz-Gaspà ${ }^{1}$, Maria C. Cid ${ }^{2}$, Ana Monegal', Núria Guañabens ${ }^{1}$, Pilar Peris ${ }^{1} .{ }^{1}$ Hospital Clinic, University of Barcelona, Metabolic Bone Diseases Unit. Department of Rheumatology, Barcelona, Spain; ${ }^{2}$ Hospital Clínic, University of Barcelona, Institut d'Investigacions Biomèdiques August Pi i Sunyer (IDIBAPS), Vasculitis Research Unit, Department of Autoimmune Diseases, Barcelona, Spain; ${ }^{3}$ Hospital Clinic, University of Barcelona, Department of Nuclear Medicine, Barcelona, Spain; ${ }^{4}$ University of Barcelona, Biostatistics, Department of Basic Clinical Practice, Barcelona, Spain

Background: Glucocorticoid-induced osteoporosis (GIOP) is the one of the most common forms of secondary osteoporosis (OP). Fractures in GIOP frequently occur with higher than expected bone mineral density (BMD) values. The Trabecular Bone Score (TBS) is a gray-level textural index derived from DXA images that provides information about bone microarchitecture and fracture risk independently of BMD; therefore, TBS measurement could be useful for identifying patients with high fracture risk associated with glucocorticoid (GC) treatment.

Objectives: To analyse the clinical utility of TBS for fracture risk assessment in GC treated patients and compare it with BMD assessment, the gold-standard diagnostic test.

Methods: 127 patients on chronic GC treatment $(\geq 5 \mathrm{mg} /$ day) were included (mean age $62 \pm 18$ years, $63 \%$ women) in this cross-sectional study. The medical history and anthropometric data were collected, as well as measurements of bone metabolism parameters, bone densitometry (DXA) at lumbar spine and femur (considering OP when T-score $\leq-2.5$ ) TBS (considering degraded microarchitecture [DMA] with values <1.230) and dorsolumbar $\mathrm{X}$-ray to assess vertebral fractures (VF). BMD and TBS 
sensitivity, specificity and positive and negative predictive values (PPV, NPV) were evaluated to determine the diagnostic accuracy of the two methods.

Results: Most of the patients were receiving GC treatment for vasculitis or polymyalgia rheumatica over a mean period of $47.7 \pm 69$ months at a mean daily dose of $14.5 \mathrm{mg} .17 \%$ had VF, $28 \%$ any type of fragility fracture (VF + no-VF), 29\% OP and 71\% DMA. In patients with VF, low TBS (DMA) was more common than densitometric OP $(76 \%, p=0.03$ vs. $38 \%, p=n . s)$. Similar results were observed when analysing patients with any fragility fracture $(69 \%, p=0.02$ vs. $36 \%, p=n . s)$. The diagnostic accuracy of TBS was greater than BMD on evaluating VF, with a sensitivity, specificity, PPV and NPV of $0.76,0.53,0.25$ and 0.92 for TBS and $0.38,0.72,0.22$, and 0.85 for BMD, respectively. Specificity increased to 0.89 for VF and 0.9 for any fragility fracture on combining both assessments (OP+DMA).

Conclusion: TBS has greater discriminative power than BMD measurement and could be useful as a complementary tool for fracture risk assessment in GIOP.

Disclosure of Interests: Helena Florez: None declared, José HernándezRodríguez : None declared, Africa Muxi: None declared, Josep Lluís Carrasco: None declared, Sergio Prieto-González: None declared, Silvia RuizGaspà : None declared, Maria C. Cid Grant/research support from: Kiniksa Pharmaceuticals, Consultant for: Roche, GSK, Janssen, Abbvie, Speakers bureau: Boehringer-Inhelheim, Vifor, Ana Monegal Speakers bureau: Eli Lilly, Amgen, Núria Guañabens Consultant for: Advisory Boards from Amgen, Alexion and UCB, Speakers bureau: Fees and lectures from Eli Lilly, Pilar Peris Speakers bureau: Personal Fees and Non-financial support (attendance to congresses) from Amgen and Eli Lilly

DOI: 10.1136/annrheumdis-2019-eular.2287

\section{FRI0488 ROLE OF DUAL-PHOTON X-RAY ABSORPTIOMETRY (DXA) IN PREDICTION OF REFRACTURE AND MORTALITY}

Sara Ganhão ${ }^{1}$, Miguel Guerra ${ }^{2}$, Francisca Aguiar ${ }^{1}$, Raquel Ferreira ${ }^{1}$, Teresa Martins-Rocha ${ }^{1}$, Ana Filipa Águeda ${ }^{3}$, Georgina Terroso ${ }^{1}$, Eva Mariz ${ }^{1}$ Carlos Vaz ${ }^{1,4}$, Lúcia Costa ${ }^{1} .{ }^{1}$ Centro Hospitalar e Universitário de São João, Department of Rheumatology, Oporto, Portugal; ${ }^{2}$ Centro Hospitalar de Vila Nova de Gaia/Espinho, Department of Rheumatology, Vila Nova de Gaia, Portugal; ${ }^{3}$ Centro Hospitalar do Baixo Vouga, Department of Rheumatology, Aveiro, Portugal; ${ }^{4}$ Faculty of Medicine of Oporto University, Oporto, Portugal

Background: The presence of a fragility fracture leads to a high risk for a subsequent fracture and mortality. Although bone mineral density (BMD) does not reflect the real fracture risk in many conditions, it is a wellestablished predictor of fracture risk and for many years, dual-photon Xray absorptiometry (DXA) has been the reference standard for measuring BMD.

Objectives: To evaluate the predictive significance of femur BMD and Tscore obtained by DXA in the outcomes refracture and mortality of elderly patients with a previous fragility hip fracture.

Methods: Longitudinal retrospective study of patients referred to the Rheumatology department's Fracture Liaison Service (FLS) from march 2015 until march 2017 aged $\geq 65$ years old. Demographic and clinical data were collected including DXA results. Statistical analysis was performed with STATA. Hazard ratios (HR) were calculated through cox regression, adjusted to age and degree of dependence.

Results: From a total of 522 patients, 214 performed DXA (77.6\% female; median (min-max) age 82 (65-99) years; $65.4 \%$ autonomous). Twenty seven patients died (median days until death=457, min 160, max 1049) and 18 had a subsequent fracture (median days until refracture 343.5, $\min 160, \max 1049) ; 129$ patients maintained follow-up. Mean serum total femur BMD, $\mathrm{g} / \mathrm{cm}^{2}$ (SD) was 0.7 (0.14) and mean total femur T-score (SD) was -2.6, (1.1). 127 (59.3\%) patients had T-score $\leq-2.5$. After multivariable analysis, higher femur BMD and T-score were predictors of better survival outcome (HR 0.01, $p=0.023$; HR 0.67, $p=0.038$, respectively). Nevertheless, none of them predicted refracture outcome. (HR: 0.08; $95 \% \mathrm{Cl} \quad[0.01-5.16] ; p=0.240) ; \mathrm{HR}: 0.81 ; 95 \% \mathrm{Cl}[0.51-1.28]$; $p=0.361$ ).

Conclusion: DXA is important in the management of patients with bone fragility syndromes. Our sample suggests that femur BMD/T-score had significant impact on survival outcome. Thus it can be a predictor of mortality with relevant role in daily practice.

\section{REFERENCES:}

[1] Browner WS, Seeley DG, Vogt TM, Cummings SR. Non-trauma mortality in elderly women with low bone mineral density. Study of Osteoporotic Fractures Research Group. Lancet 1991;338:355-8.

[2] Trivedi DP, Khaw KT. Bone mineral density at the hip predicts mortality in elderly men. Osteoporos Int 2001;12:259-65.

[3] Cortet B. Does low bone mineral density predict mortality? Joint Bone Spine. 2016 Dec;83(6):623-624

[4] Roux $\mathrm{C}^{1}$, Briot $\mathrm{K}^{2}$. Current role for bone absorptiometry. Joint Bone Spine. 2017 Jan;84(1):35-37.

[5] Cummings SR, Black DM, Nevitt MC, Browner W, Cauley J, Ensrud K,et al. Bone density at various sites for prediction of hip fractures. The Study of Osteoporotic Fractures Research Group. Lancet. 1993:341:72-75.

Disclosure of Interests: None declared

DOI: 10.1136/annrheumdis-2019-eular.5066

\section{FRI0489 THE ASSOCIATION BETWEEN CT-MEASURED BONE ATTENUATION AND PREVALENT VERTEBRAL FRACTURES IN THE THORACIC SPINE DIFFERS ACCORDING TO VERTEBRAL FRACTURE LOCATIONS}

Mayke J van Dort ${ }^{1}$, Annemariek Driessen ${ }^{2}$, Joop van den Bergh ${ }^{1}$, Emiel

Fm Wouters ${ }^{1}$, Elisabeth AM Romme ${ }^{3}$, Frank Wjm Smeenk ${ }^{3}$, Bert van Rietbergen ${ }^{4}$ Piet Geusens ${ }^{1} .{ }^{1}$ Maastricht University, Maastricht, Netherlands; ${ }^{2}$ Maastrcht

University, Maastyricht, Netherlands; ${ }^{3}$ Catharina Hospital, Eindhoven, Netherlands; ${ }^{4}$ TU Eindhoven, Eindhoven, Netherlands

Background: Subjects with prevalent vertebral fractures (VFs) have lower bone mineral density (BMD) in the lumbar spine and hip than subjects without prevalent VFs. However, VFs are not equally distributed across the thoracic spine. They occur preferentially at T7-T8 and T11-T12, which are the highest loaded vertebral regions during daily activities (1).

Objectives: We evaluated the association of BMD with prevalent VFs at non-preferential locations (nprVFs: T4-T6 and T9-T10) and at preferential locations (prVFs: T7-T8 and T11-T12).

Methods: Baseline CT images of T4-T12 in smokers with and without COPD were analysed for the presence of VFs according to Genant Bone attenuation (BA), expressed in Hounsfield units (HU), was measured in all non-fractured vertebrae. Linear regression was used to compare mean BA and logistic regression was used to estimate the association between BA and prevalent VFs. All analyses were adjusted for age and sex.

Results: Prevalent VFs were most frequently located at T7-T8 (in $>6 \%$ of vertebrae) and $\mathrm{T} 11-\mathrm{T} 12$ (in $>4 \%$ of vertebrae).

At subject level and compared to subjects without prevalent VFs, mean BA of all non-fractured vertebrae was significantly lower in subjects with any prevalent VFs $(-21 \%, p<0.0001)$. There was a significant progressive trend $(p<0.0001)$ in decrease in mean BA of all non-fractured vertebrae between subjects with only prVFs $(-15 \%)$, with only nprVFs $(-25 \%$, $p<0.05$ vs prVFs) and with both nprVFs and prVFs combined $(-32 \%$, $p<0.0001$ vs prVFs) (all $p<0.0001$ compared to subjects without VF). At each individual non-fractured vertebral level, each BA decrease of 50 $\mathrm{HU}$ was associated with a 2.0-2.5 increased odds of any prevalent VF, a 1.5-1.9 increased odds of only prVF, a 2.2-3.4 increased odds of only nprVF and a 3.8-4.6 increased odds of nprVF and prVF combined.

Conclusion: In addition to BA, other factors such as vertebral load during daily activities may determine the location of a VF. The location of a VF may be a potential in vivo marker of the load/strength ratio of bone.

\section{REFERENCES:}

[1] Bruno AG, Burkhart K, Allaire B, Anderson DE, Bouxsein ML. Spinal Loading Patterns From Biomechanical Modeling Explain the High Incidence of Vertebral Fractures in the Thoracolumbar Region. J Bone Miner Res. 2017 Jun;32(6):1282-1290

Disclosure of Interests: Mayke J van Dort: None declared, Annemariek Driessen: None declared, Joop van den bergh Grant/research support from: Grants/research support from Eli Lily, Sanofi, Amgen., Emiel FM Wouters: None declared, Elisabeth AM Romme: None declared, Frank WJM Smeenk: None declared, Bert van Rietbergen: None declared, Piet Geusens Grant/research support from: Research support, consultant and/ or speaker fees from: Pfizer, Abbott, Eli Lilly, Amgen, MSD, Roche, UCB, BMS, Novartis, Will-Pharma, Grant/research support from: Pfizer, 